Running head: Estimation performance in an atypical number line task

\title{
Benchmark-based Strategy Use in Atypical Number Lines
}

\author{
Koen Luwel ${ }^{1,2}$, Dominique Peeters ${ }^{2}$, Goedele Dierckx ${ }^{1}$, Elke Sekeris ${ }^{2}$, and Lieven Verschaffel ${ }^{2}$ \\ ${ }^{1}$ Centre for Mathematics, Education, Econometrics and Statistics, KU Leuven - Campus \\ Brussels, Belgium \\ ${ }^{2}$ Centre for Instructional Psychology and Technology, KU Leuven, Belgium
}

\section{Author Notes:}

This study was supported by grant GOA 2012/010 of the Research Fund KU Leuven, Belgium. The authors would like to thank Ismene Weerelds for her help in data collection as well as the two anonymous reviewers for their helpful comments on an earlier version of this paper .

The raw data from this study are available at https://osf.io/gzytb/.

Correspondence concerning this article should be addressed to Koen Luwel, Centre for Mathematics, Education, Econometrics and Statistics, KU Leuven - Campus Brussels Warmoesberg 26, B-1000 Brussels, Belgium.

E-mail: koen.luwel@kuleuven.be 


\begin{abstract}
Previous studies have indicated that the presence of atypical endpoints (e.g., 1,639 and 2,897 ) on a number line has a negative effect on number line estimation (NLE) performance (Booth \& Newton, 2012; Hurst et al., 2014). In the present study, we investigated whether this effect could be attributed to a disruption in the ease with which benchmarks on the number line can be determined and whether this possible disruption changes with age. Hence, we asked fifth graders and adults to perform a NLE task in a typical and an atypical condition with a number line ranging from 0 to 1,000 and from 367 to 1,367 , respectively. Results showed that participants' overall estimates were less accurate in the atypical condition compared to the typical condition. A similar pattern of results was observed for the estimates around the self-created benchmarks at $50 \%$ in children and at 25, 50, and $75 \%$ in adults. Moreover, in both age groups, we found a shift from a larger proportion of estimation patterns being best fit by more complex power models in the typical condition towards a larger proportion of estimation patterns best described by simpler power models in the atypical condition. Importantly, we also found evidence that adults' estimation patterns in the typical condition are best described by a fourcycle power model, hereby extending previous modelling results. Overall, our findings indicate that hindering the application of benchmark-based strategies negatively affects children's and adults' NLE performance.
\end{abstract}

Key words: Number line estimation; strategy use; atypical endpoints; benchmarks; cyclic power model

Public Significance: Fifth graders and adults were asked to estimate the position of a set of numbers on two different number lines: a 0-1000 (typical) and a 367-1367 (atypical) number line. It was found that the inclusion of atypical endpoints had a negative effect on number line estimation performance. This impoverished performance can be attributed to hindering the use of 
refined benchmark-based strategies, because the mental calculations needed to create or use the internal benchmarks were more difficult. Taken together, these findings add new evidence that number line estimation performance can at least be partly explained by the application of benchmark-based strategies 
During the last decade, research on number line estimation (NLE) has expanded rapidly. In a typical NLE task, an empty number line with labelled endpoints (e.g., 0 and 1,000) is presented and participants are asked to estimate the spatial position of a given number on that line. One recurrent finding across various studies on NLE is that estimation performance improves with age (e.g., Berteletti, Lucangeli, Piazza, Dehaene, \& Zorzi, 2010; Booth \& Siegler, 2006; 2008; Siegler \& Booth, 2004). Initially, some researchers claimed that these age-related improvements can be explained by a shift from an underlying logarithmic mental magnitude representation towards a linear one (e.g., Booth \& Siegler, 2006; Laski \& Siegler, 2007; Siegler \& Booth, 2004; Siegler \& Opfer, 2003). Underlying this claim is the assumption that the shape of people's estimation patterns allows for direct inferences about the nature of their spatial representation of numerical magnitude.

Recently, however, it has been argued that people rely on benchmarks when making NLEs, and that improvement in NLE performance is at least partly due to developmental changes in the application of benchmark-based strategies. Different sources of evidence in favour of such benchmark-based strategy use in NLE have been raised: (a) the superior fittings of one- and twocycle power functions compared to logarithmic and linear functions on individuals' estimation patterns (Barth \& Paladino, 2011; Barth et al., 2016; Cohen \& Blanc-Goldhammer, 2011; Reinert, Huber, Nuerk, \& Moeller, 2015; Rouder \& Geary, 2014; Slusser, Santiago, \& Barth, 2013; though see Opfer, Thompson, \& Kim, 2016), (b) participants' error rates and estimation latencies (Ashcraft \& Moore, 2012), (c) participants' verbal reports and observations of their solution behaviour (Newman \& Berger, 1984; Peeters, Degrande, Ebersbach, Verschaffel, \& Luwel, 2016; Peeters, Verschaffel, \& Luwel, 2017; Petitto, 1990), and, (d) eye-movement data (Heine et al., 2010; Schneider et al., 2008; Sullivan, Juhasz, Slattery, \& Barth, 2011). 
Based on the evidence coming from all these sources, the development in children's benchmark-based strategy use in a typical NLE task can be depicted generally as follows. First, it evolves from using one external benchmark at the origin to relying on both external benchmarks at the origin and endpoint. Later on, children make use of both external benchmarks and a selfcreated, internal benchmark at the midpoint to perform their estimates (Ashcraft \& Moore, 2012; Barth \& Paladino, 2011; Newman \& Berger, 1984; Peeters et al., 2017; submitted; Petitto, 1990; Schneider et al., 2008; Slusser et al., 2013; Xu \& LeFevre, 2016). Recently, it has been shown that children also rely on internal benchmarks at the quartiles (i.e., 25 and $75 \%$ ) of the number line in addition to using benchmarks at the origin, midpoint, and endpoint (Peeters et al., submitted), albeit to a lesser extent than adults do (Peeters et al., 2017). These shifts from relying on one benchmark at the origin to using more, and more refined, benchmarks are accompanied with improvements in NLE performance (e.g., Ashcraft \& Moore, 2012, Barth \& Paladino, 2011; Newman \& Berger, 1984; Peeters et al., 2017, submitted; Slusser et al., 2013).

The application of strategies that are based on such internal benchmarks involves the complex interplay of spatial, numerical, and arithmetic competencies (e.g., Link, Nuerk, \& Moeller, 2014; Simms, Clayton, Cragg, Gilmore, \& Johnson, 2016). First, one has to infer which benchmark is closest to estimate from and then parse the number line into two or four segments to position the midpoint and quartiles, respectively. Next, one has to derive the numerical values that correspond to these positions (e.g., knowing that the midpoint on a 0 and 1,000 number line corresponds with 500) and decide whether the target number is smaller or larger than the benchmark. Finally, one has to further refine the estimated position of the target number in relation to the benchmark (e.g., if 500 is here then 493 is just a little bit more to the left).

Based on the above-mentioned rational task analysis, it can be expected that, when mental calculations needed to create or use the internal benchmarks become more difficult, individuals 
might refrain from using strategies based on these benchmarks. For example, on a number line with atypical endpoints such as 367 and 1,367, it is more difficult to calculate the corresponding values of the internal benchmarks at the midpoint and quartiles than for a typical number line bounded by two round values such as 0 and 1,000. To the best of our knowledge, only two studies have included such atypical number lines. Booth and Newton (2012) compared the linearity of the estimation patterns of middle school students on a typical $0-10,000$ with an atypical 0 to 6,257 number line. They found that the estimation pattern for the atypical number line $\left(R^{2} \operatorname{Lin}=.85\right)$ was significantly less linear than for the typical one $\left(R^{2} \operatorname{Lin}=.96\right)$. Similarly, Hurst, Leigh Monahan, Heller, and Cordes (2014) presented adults with three types of number lines: a typical number line ranging from 2,000 to 3,000, a number line with one atypical endpoint value ranging from 0 to 1,258, and a number line with a similar range of 1,258 but with two atypical endpoints ranging from 1,639 to 2,897. Their results indicated that the estimation pattern of the $1,639-2,897$ number line was significantly better fit by a logarithmic $\left(R^{2} \log =.99\right)$ than by a linear model $\left(R^{2}\right.$ Lin $\left.=.98\right)$, while the opposite was the case for the $2,000-3,000\left(R^{2} \log =\right.$ .97 vs. $\left.R^{2} \operatorname{Lin}=.98\right)$ and $0-1,258$ number line $\left(R^{2} \log =.73\right.$ vs. $\left.R_{\text {Lin }}^{2}=1.00\right)$.

Although both studies hypothesized that the use of atypical number lines would make the application of benchmark-based strategies more difficult, if not impossible, they do not provide any direct evidence that helps to explain the observed negative impact of these atypical number lines on the linearity of participants' NLE patterns in terms of participants' impaired benchmark use. In the present study, we examined the extent to which number lines with typical endpoints would negatively affect the application of estimation strategies based on internal benchmarks by directly comparing participants' estimation accuracy and estimation patterns in a typical and atypical NLE task. More precisely, participants had to make NLEs in three conditions that all involved a number line with a range of 1,000 but differed in terms of their origin and endpoint. 
The typical condition involved a 0 to 1,000 , the atypical a 367 to 1,367 , and the control condition a 1,000 to 2,000 number line. The latter condition was included to rule out the possibility that the potentially weaker estimation performance in the atypical condition might be merely due to the use of larger magnitudes rather than to the inclusion of unusual endpoints. In addition, since it is reasonable to assume that the spatial, numerical, and arithmetical skills employed to calculate and use the internal benchmarks develop with age, we examined whether the negative effect of the atypical endpoints was more pronounced in children than in adults. Hurst et al. (2014), for instance, found only very small differences between conditions with respect to adults' $R^{2}$ Lin, suggesting that adults were still capable of using benchmark-based estimation strategies, at least to some extent.

Three sets of hypotheses were tested. Firstly, regarding overall estimation accuracy, we hypothesized that participants would estimate less accurately in the atypical condition than in the typical condition because the use of atypical endpoints makes it more difficult to determine and use internal benchmarks at 25,50 , and $75 \%$ of the number line, hereby negatively affecting their NLE performance. Furthermore, we expected that this negative effect of the atypical endpoints would be more pronounced in children than in adults.

Secondly, we predicted that especially target numbers near 25,50 , and $75 \%$ of the number line would be estimated less accurately in the atypical compared to the typical condition, because determining and using internal benchmarks at the midpoint and quartiles is more difficult in number lines with atypical endpoints. Again, we expected that this difference would be more pronounced in children than in adults. No difference was anticipated between conditions for target numbers in the neighbourhood of 0 and $100 \%$ of the number line, since these benchmarks did not need to be determined by the participants. 
Finally, as has been demonstrated by Barth and colleagues (Barth \& Paladino, 2011; Slusser et al., 2013) the use of benchmarks on the number line is reflected in participants' estimation patterns. This benchmark use can be identified by fitting $n$-cycle power functions on the individual estimation patterns. Since children as young as third grade use strategies based on an internal benchmark at the midpoint (e.g., Ashcraft \& Moore, 2012), we predicted that most fifth graders' estimates in the typical condition would be best fit by a two-cycle model, reflecting the strategic use of the origin, endpoint, and midpoint when making estimates. Similarly, a minority of fifth graders have been observed to rely on quartiles too (Peeters et al., submitted), therefore these children's estimates would be best fit by a four-cycle power model, indicating the use of strategies based on the origin, midpoint, quartiles, and endpoint. In contrast, children's estimates in the atypical condition would be best described by a one-cycle model, reflecting the strategic use of strategies based on the origin and endpoint only. Adults' estimates in the typical condition would be best fit by a four-cycle power model as they can rely on the quartiles too, whereas their estimates in the atypical condition would be best described by a one- or a two-cycle model.

\section{Method}

\section{Participants}

Thirty fifth-grade children $(M=10$ yrs., $S D=0.42$ yrs., 17 girls $)$ from a primary school in Flanders, Belgium, and 32 higher education students $(M=22$ yrs., $S D=1.50$ yrs., 28 girls $)$ participated in the experiment. The children's parents and the adult participants had provided written informed consent before the start of the experiment, and at the start participants were told that they could quit the experiment at any moment.

\section{Materials and Procedure}


Participants had to perform the NLE task in three conditions (see Figure 1). In the typical condition, they were presented with number lines bounded by 0 at the origin and 1,000 at the endpoint. In the atypical condition, they were given number lines with 367 at the origin and 1,367 at the endpoint. Finally, in the additional control condition, number lines bounded by 1,000 at the origin and by 2,000 at the endpoint were provided. To enable a proper comparison between conditions, all three conditions had a range of 1,000 . The order of the conditions was randomized across participants. For each target number, a new number line with a length of $22 \mathrm{~cm}$ was presented on a separate sheet of white paper. In all three conditions, participants had to position 20 target numbers on the number line, the order of which was randomized across participants. The target numbers were randomly selected with the following restrictions: (a) to prevent overestimation of smaller numbers due to oversampling at this end of the number line (Ebersbach, Luwel, Frick, Onghena, \& Verschaffel, 2008), we ensured that the numbers were equally distributed across the number line by selecting two numbers from each hundred between 0 and 1,000, and (b) every unit, decade, and hundred appeared equally often. Furthermore, to maximize comparability between the three conditions, the relative positions of the target numbers were matched, namely at $\underline{0.5}, 6.9,12.3,19, \underline{24.6}, 27.1,33.7,38.4,42.2,45.8, \underline{50.2}, 57.3,61.6$, $66.4,71, \underline{75.5}, 83.9,88.8,94.1$, and $\underline{99.7} \%$ of the number line (see Appendix 1). The underlined positions refer to five target numbers that were intentionally located close to the position of a potential benchmark (i.e., at $0,25,50,75$, and $100 \%$ of the number line). Before estimating the target numbers in each condition, participants received two practice numbers, one at 40.8 and another at $85.1 \%$ of the number line (see Appendix 1).

Participants were tested individually in a quiet room, either at their school or in a room located at the university. The instructions were exactly the same for the two age groups: Each participant was asked to put a mark on the line where he/she thought the target number would go. 
Participants were only provided with a pencil. They were not provided with feedback during the experiment. Completion of the three conditions altogether lasted on average about 40 minutes per child and about 30 minutes per adult.

(Figure 1 about here)

\section{Model Fittings}

To model the individual NLE patterns, $n$-cycle power functions have been used to detect the possible use of benchmarks. The use of these cycle power models is based on the assumption that that number line estimation task should be regarded as a proportion judgment task (Barth \& Paladino, 2011; Slusser et al., 2013). According to this proportion judgement account, a given target number $x$ on a number line bounded by two benchmarks $b_{\min }$ and $b_{\max }$,can be positioned by assessing the proportion $p=\left(x-b_{\min }\right) /\left(b_{\max }-b_{\min }\right)$ of the smaller magnitude $x-b_{\min }$ to the the total length of the number line $b_{\max }-b_{\min }$. As such, the participant will position the target number $x$ at:

$$
b_{\min }+p\left(b_{\max }-b_{\min }\right)
$$

Spence (1990), followed by Hollands et al. (2000) and Slusser et al.(2013) applied Stevens' power law to estimate an actual magnitude $m$ by $\tilde{\alpha} m^{\beta}$, where $\tilde{\alpha}$ is a scaling factor and $\beta$ is the exponent of the power function. According to this law $p=\left(x-b_{\min }\right)^{\beta} /\left(\left(x-b_{\min }\right)^{\beta}+\alpha\left(b_{\max }-x\right)^{\beta}\right)$. This equation extends the previous work of Slusser et. al (2013) where $\alpha$ is set to 1.

When a participant solves the NLE task by only using the two endpoints $b_{0}$ and $b_{100}$ of the number line, then equation (1) is applied to position a target number $x$ using the two endpoints of 
the number line as $b_{\min }$ and $b_{\max }$. The resulting equation is the model equation of the one-cycle power model:

$$
\mathrm{b}_{0}+\left(\mathrm{b}_{100}-\mathrm{b}_{0}\right)\left(x-\mathrm{b}_{0}\right)^{\beta} /\left(\left(x-\mathrm{b}_{0}\right)^{\beta}+\alpha\left(\mathrm{b}_{100}-\mathrm{x}\right)^{\beta}\right)
$$

The two-cycle model is used when in addition to the two endpoints $b_{0}$ and $b_{100}$, the midpoint $\mathrm{b}_{50}$ is used as a benchmark. The model equation can be obtained by dividing the number line in half and applying the abovementioned strategy twice, once to the first half and once to the second half. According to the two-cycle model, the participant will position the target number $x$ at

$$
\begin{aligned}
& b_{0}+\left(b_{50}-b_{0}\right)\left(x-b_{0}\right)^{\beta} /\left(\left(x-b_{0}\right)^{\beta}+\alpha\left(b_{50}-x\right)^{\beta}\right) \text { if } x<b_{50} \\
& b_{50}+\left(b_{100}-b_{50}\right)\left(x-b_{50}\right)^{\beta} /\left(\left(x-b_{50}\right)^{\beta}+\alpha\left(b_{100}-x\right)^{\beta}\right) \text { if } x>b_{50}
\end{aligned}
$$

Moreover, to identify participants who also used the quartiles as benchmarks, we included a four-cycle power model, in which the target number $x$ is estimated to be at the following position

$$
\begin{array}{ll}
\mathrm{b}_{0}+\left(\mathrm{b}_{25}-\mathrm{b}_{0}\right)\left(x-\mathrm{b}_{0}\right)^{\beta} /\left(\left(x-\mathrm{b}_{0}\right)^{\beta}+\alpha\left(\mathrm{b}_{25}-x\right)^{\beta}\right) & \text { if } \mathrm{b}_{0}<x<\mathrm{b}_{25} \\
\mathrm{~b}_{25}+\left(\mathrm{b}_{50}-\mathrm{b}_{25}\right)\left(x-\mathrm{b}_{25}\right)^{\beta} /\left(\left(x-\mathrm{b}_{25}\right)^{\beta}+\alpha\left(\mathrm{b}_{50}-x\right)^{\beta}\right) & \text { if } \mathrm{b}_{25}<x<\mathrm{b}_{50} \\
\mathrm{~b}_{50}+\left(\mathrm{b}_{75}-\mathrm{b}_{50}\right)\left(x-\mathrm{b}_{50}\right)^{\beta} /\left(\left(x-\mathrm{b}_{50}\right)^{\beta}+\alpha\left(\mathrm{b}_{75}-x\right)^{\beta}\right) & \text { if } \mathrm{b}_{50}<x<\mathrm{b}_{75} \\
\mathrm{~b}_{75}+\left(\mathrm{b}_{100}-\mathrm{b}_{75}\right)\left(x-\mathrm{b}_{75}\right)^{\beta} /\left(\left(x-\mathrm{b}_{75}\right)^{\beta}+\alpha\left(\mathrm{b}_{100}-x\right)^{\beta}\right) & \text { if } \mathrm{b}_{75}<x<\mathrm{b}_{100}
\end{array}
$$

The technical details of the different model equations can be found in Appendix 2.

The Akaike Information Criterion corrected for small samples (AICc) was used to determine which of the abovementioned models could best explain participants' estimation patterns (Barth \& Paladino, 2011). This measure takes into account goodness of fit and model complexity (i.e., number of parameters) whereby a lower AICc value refers to a better model fit. Differences in AICc scores (i.e., $\triangle \mathrm{AICc}$ ) reflect the amount of support for one specific model in comparison to the other models. According to Burnham and Anderson (2002), models having a 
$\triangle \mathrm{AICc}$ within $0-2$ of the best model have substantial support and should be taken into consideration when making inferences, models with a $\triangle \mathrm{AICc}$ within 4-7 have considerably less support, and models with a $\triangle \mathrm{AICc}>10$ have essentially no support.

\section{Results}

Accuracy of number line estimates was determined by calculating the percentage absolute error (PAE; Booth \& Siegler, 2006). For children and adults separately and for each of the three conditions, individual estimates that deviated more than $2 S D$ s from participants' mean estimate were excluded. For the children, 85 estimates out of a total of 1,800 were removed (i.e., 5\%, of which 35 in the typical, 23 in the atypical, and 27 in the control condition). For the adults, 104 estimates out of a total of 1,920 were removed (i.e., 5\%, of which 31 in the typical, 36 in the atypical, and 37 in the control condition). Throughout the analyses, Tukey HSD tests were used in all post hoc comparisons.

\section{Overall Accuracy}

In a first analysis, we looked whether overall accuracy was different dependent on participants' age as well as on the type of NLE task. We therefore ran a 2 (Age: children vs. adults) $\times 3$ (Condition: typical, atypical, control) ANOVA with repeated measures on the last variable and PAE as dependent variable. This analysis revealed a significant main effect of age, $F(1,60)=126.68, p<.0001$, and condition, $F(2,120)=23.33, p<.0001$. Furthermore, a significant interaction, $F(2,120)=6.74, p=.002$, was found (see Figure 2). The interaction effect revealed that children estimated, as expected, significantly more accurately in the typical $(M=6.47 \%, S D=1.92 \%)$ compared to the atypical condition $(M=9.11 \%, S D=2.87 \%, p=$ $.0001)$. For adults, this difference was only marginally significant $(p=.10)$. They tended to estimate more accurately in the typical $(M=3.26 \%, S D=0.87 \%)$ than in the atypical condition $(M=4.27 \%, S D=1.03 \%)$. 
To rule out the possibility that the weaker estimation performance in the atypical condition might be due to the use of larger magnitudes rather than to inclusion of unusual endpoints, we compared performance in the typical condition with performance in the control condition. As can be derived from the significant interaction, no differences were found between the typical $(M=6.47 \%, S D=1.92 \% ; M=3.26 \%, S D=0.87 \%)$ and the control condition $(M=$ $6.87 \%, S D=2.19 \% ; M=3.88 \%, S D=1.15 \%$ ), for children and adults respectively (all $p$ s $>.10$ ). We can therefore conclude that participants' poorer estimation performance in the atypical compared to the typical condition cannot be attributed to the larger magnitudes of the target numbers in this condition, since the estimates in the control condition - which contained magnitudes that were even larger than in the 367-1,367 range - were estimated equally accurately as those in the control condition. Given the specific purpose of the control condition in the design of the study, the data from this condition will not be taken into account in the remainder of the analyses.

(Figure 2 about here)

\section{Contour Accuracy}

A contour analysis (Ashcraft \& Moore, 2012) was conducted to assess whether estimation accuracy at $0,25,50,75$, and $100 \%$ of the number line differed between the typical and atypical condition. We conducted a 2 (Age: children vs. adults) $\times 2$ (Condition: typical vs. atypical) $\times 5$ (Location of benchmark: 0, 25, 50,75, and 100\%) ANOVA with repeated measures on the last two variables and PAE as dependent variable.

Results indicated a main effect for age, $F(1,60)=109.38, p<.0001$, condition, $F(1,60)=$ 57.13, $p<.0001$, and location, $F(4,240)=53.67, p<.0001$. Furthermore, a significant Age $\times$ 
Condition, $F(1,60)=8.47, p=.005$, Age $\times$ Location, $F(4,240)=4.37, p=.002$, Condition $\times$ Location, $F(4,240)=10.22, p<.0001$, and Condition $\times$ Location $\times$ Age interaction, $F(4,240)=$ $4.34, p=.002$, was found (see Figure 3 and Table 1). When resolving the latter three-way interaction for age, we observed that children estimated equally accurately at $0,25,75$, and $100 \%$ of the number line in the typical and atypical condition. However, at 50\% of the number line, children's estimates were significantly more accurate in the typical condition in comparison to the atypical condition $(p=.0002)$. Adults estimated equally accurately at 0,25 , and $100 \%$ in the typical and atypical condition, but significantly more accurately at 50 and $75 \%$ of the number line in the typical than in the atypical condition (all $p \mathrm{~s}=.0004$ ).

We also compared the PAE at the different benchmarks locations for each condition and age group. For the typical condition, children were significantly more accurate at the origin than at 25\% ( $p=.009)$ and $75 \%(p=.001)$ of the number line. A similar pattern of results was observed for the adults: they were significantly more accurate at the origin than at $25 \%$ ( $p<$ $.0001)$ and $75 \%(p=.04)$ of the number line. In addition, they were significantly more accurate at the endpoint than at $25 \%(p<.0001)$ of the number line. For the atypical condition, children were significantly less accurate at $25 \%$ than at the endpoint $(p=.002)$ and significantly less accurate at $50 \%$ than at the origin $(p=.0002), 25 \%(p=.01)$, and the endpoint $(p=.0002)$, and significantly less accurate at $75 \%$ than at the origin $(p=.02)$ and the endpoint $(p=.0002)$ of the number line. Adults were significantly less accurate at $25 \%, 50 \%$, and $75 \%$ of the number line compared to the origin and endpoint (all $p \mathrm{~s}<.0001$ ).

(Figure 3 and Table 1 about here)

\section{Estimation Patterns}


To test whether the kind of benchmark-based strategies used was reflected in participants' estimation patterns, we fitted a one-, a two-, and a four-cycle power model on children's and adults' individual estimation patterns in the typical and atypical condition (see Table 2). We conducted, for each age group separately, a Wilcoxon Signed Rank Test whereby the type of model was treated as an ordinal variable (i.e., best fit with the 1-cycle model was considered as the lowest category and best fit the 4-cycle model was considered as the highest category). This test revealed, for the adult participants, a significant difference between both conditions with respect to the distribution of participants across the three models, $z=2.10, p=.04, r=.37$. It was found that more participants were best described by a more advanced model in the typical compared to the atypical condition $(\mathrm{T}=15$; all $\Delta \mathrm{AICc}>100)$. We did not observe a significant difference between both conditions for the children, although results tended to indicate a shift in the proportion of children's estimation patterns' best fit by the two-cycle model towards the onecycle model from the typical to the atypical condition (all $\triangle \mathrm{AICc}>130$ ).

In addition to these analyses on the individual level, analyses on the group level were conducted by fitting these three models on the median estimates of children and adults for the typical and atypical condition (see Appendix 3). We have included the results of these analyses to allow for a direct comparison with the results of other studies (e.g., Ashcraft \& Moore, 2012; Barth \& Paladino, 2011; Slusser et al., 2013) that fitted cyclical power models to group-based estimation patterns. However, given that these median estimation patterns consist of a collection of data points originating from different participants whose individual estimation patterns can differ from each other in terms of the best fitting model, one should be cautious in making strong inferences from such analyses at the group level (Firebaugh, 2015).

(Table 2 about here) 


\section{Discussion}

Previous studies have indicated that using number lines with atypical endpoints has a negative impact on the linearity of participants' NLE patterns (Booth \& Newton, 2012; Hurst et al., 2014). In the present study, we investigated whether this observation was due to an increased difficulty with which internal benchmarks on the number line could be determined and used and whether this changes with age. We asked fifth graders and adults to perform a NLE task in a typical, an atypical, and an additional control condition with number lines ranging from 0 to $1,000,367$ to 1,367 , and 1,000 to 2,000 , respectively. Importantly, we also investigated whether children's and adults' estimation patterns in the typical condition might be best described by a four-cycle power model.

As expected, children and adults estimated less accurately in the atypical condition compared to the typical condition. Importantly, this weaker estimation performance in the atypical condition was not due to the larger magnitudes of the target numbers in this condition, since the estimates in the typical condition were equally accurate as in the control condition which involved even larger magnitudes. The contour analysis of children's accuracy revealed that they were less accurate near the midpoint in the atypical than in the typical condition. A closer inspection of Figure 3 indicated that children in the typical condition exhibited a typical 'Mshaped' pattern, with a dip in errors and variability at the midpoint, suggesting that, in line with previous findings (e.g., Ashcraft \& Moore, 2012; Peeters et al., 2017, submitted; Schneider et al., 2008; White \& Szucs, 2012), they created an internal benchmark at the midpoint in addition to their use of the benchmarks at the origin and endpoint. In contrast, the PAEs in the atypical condition showed a 'tent-shaped' pattern, with small errors and variability at both the origin and endpoint, which gradually increase towards the midpoint. This latter pattern is suggestive of a strategic approach whereby children only relied on the origin and endpoint to make their 
estimates (Ashcraft \& Moore, 2012). A similar line of reasoning can be followed with respect to adults' contour accuracy, for which we also observed an M-shaped pattern for the typical condition (see Figure 3). For the atypical condition, however, the adults' pattern of results is less clear. This pattern seemed to be in between a tent-shaped and an M-shaped pattern. A visual inspection of the individual contour plots revealed that some adults exhibited an M-shaped pattern, whereas others displayed a tent-shaped pattern. Taken together, the findings from the atypical condition support the hypothesis that some adults (i.e., these that exhibited an M-shaped pattern in their contour plots) were able to apply a midpoint-based strategy in the atypical condition too, whereas the children were not able to do so.

One could alternatively hypothesize that the poorer performance at the midpoint in the atypical condition could be the result of participants having incorrectly derived the value of the midpoint, which in its turn would also lead to systematic deviations of the target values surrounding the midpoint. For instance, Luwel, Peeters, and Verschaffel (submitted) observed that some third graders were not able to correctly identify the given benchmarks at the quartiles and the midpoint of a 0-1000 number line, leading to systematic estimation errors. We therefore inspected participants' actual estimates of the target values around the midpoint but only found one child and one adult who systematically underestimated these values with a difference of about 33. This suggests that they have relied on a midpoint with a numerical value of 900 instead of 867 and thus seemed to have rounded the origin and endpoint to 400 and 1400, respectively. Whereas the findings concerning contour accuracy provide evidence for the use of specific benchmark-based strategies at a group level (i.e., data are aggregated across participants in a particular age group and condition) and might therefore obscure the specific strategy use of a particular individual, identifying the best fitting power model on the estimation data of a single participant provides more fine-grained information about the strategy use at an individual level. 
The modelling results corroborated the findings from the contour analysis. On a general level, they revealed a shift from a larger proportion of estimation patterns best fit by more complex power models in the typical condition towards a larger proportion of estimation patterns best described by simpler power models in the atypical condition. More specifically, we observed a tendency in the data that suggested a decrease in the proportion of children's estimation patterns that were best described by a two-cycle model and an increase in the proportion of children's estimation patterns that were best described by a one-cycle model from the typical to the atypical condition. According to Barth and Paladino (2011) and Slusser et al. (2013), estimation patterns best fit by a two-cycle model are considered evidence for the use of strategies based on benchmarks at the origin, midpoint, and endpoint, while a one-cycle model reflects the application of strategies based on the origin and the endpoint only. Similarly, the proportion of adults' estimation patterns best fit by the four-cycle model decreased while the proportion of adults' estimation patterns best described by a one- or a two-cycle model increased from the typical to the atypical condition. When best fit by a four-cycle model, participants use strategies based on three additional, internal benchmarks at 25,50 , and $75 \%$ of the number line in addition to applying strategies based on the two external benchmarks at the origin and the endpoint. Although most adults in the typical condition seemed to rely on three internal benchmarks at 25 , 50 , and $75 \%$, we have no clear explanation for the lack of difference between the typical and atypical condition in estimation accuracy around the first quartile.

This is, to the best of our knowledge, the first study in which a four-cycle power model has been applied to reveal that individuals apply quartile-based strategies in NLE, hereby extending previous modelling results (Barth \& Paladino, 2011; Cohen \& Blanc-Goldhammer, 2011; Rouder \& Geary, 2014; Slusser et al., 2013). It should be noted that Cohen and Blanc- 
Goldhammer (2011) already fitted a four-cycle model on adults' estimation patterns, but they found that only one participant was best fit by this four-cycle model.

Overall, our findings indicate that, when the characteristics of the task make it more difficult to generate internal benchmarks on the number line, individuals resort to less refined benchmark-based strategies. Similar patterns of results have been observed in unbounded NLE (Cohen \& Blanc-Goldhammer, 2011; Link, Huber, Nuerk, \& Moeller, 2014). In an unbounded NLE task, the labelled endpoint at the right side of the number line is missing and, instead, a scaling unit representing the distance between 0 and 1 is given together with a labelled origin. When comparing estimation performance in a bounded NLE task with performance in an unbounded NLE task, very different patterns of results emerge. For instance, in the unbounded NLE task, estimation variability increases linearly with increasing size of the target numbers while target numbers near the origin, midpoint, and endpoint are estimated with less variability in the bounded version. Similarly, estimation patterns in the unbounded task were best fit by a noncycle power model, suggesting that participants only relied on the origin of the number line to generate their estimates. In contrast, estimation patterns in the bounded task were best fit by a non-, a one-, and a two-cycle power model (see also Barth \& Paladino, 2011; Slusser et al., 2013). In light of our results, the absence of a labelled endpoint might have prevented participants to perform the mental calculations needed to create and use internal benchmarks at 25,50 , or $75 \%$ of the number line.

To conclude, our results suggest that the inclusion of atypical endpoints had a negative effect on NLE performance. Participants' impoverished NLE performance can be attributed to hindering the use of refined benchmark-based strategies, because the mental calculations needed to create or use the internal benchmarks were more difficult. Taken together, these findings add 
new evidence that age-related improvements in NLE performance is at least partly due to developmental changes in the application of benchmark-based strategies. 


\section{References}

Ashcraft, M. H., \& Moore, A. M. (2012). Cognitive processes of numerical estimation in children. Journal of Experimental Child Psychology, 111, 246-267. doi:10.1016/j.jecp.2011.08.005

Barth, H. C., \& Paladino, A. M. (2011). The development of numerical estimation: Evidence against a representational shift. Developmental Science, 14, 125-135. doi:10.1111/j.14677687.2010.00962.x

Barth, H., Slusser, E., Kanjlia, S., Garcia, J., Taggart, J., \& Chase, E. (2016). How feedback improves children's numerical estimation. Psychonomic Bulletin \& Review, 23, 11981205. doi:10.3758/s 13423-015-0984-3

Berteletti, I., Lucangeli, D., Piazza, M., Dehaene, S., \& Zorzi, M. (2010). Numerical estimation in preschoolers. Developmental Psychology, 46, 545-551. doi:10.1037/a0017887

Booth, J.K., \& Newton, K.L. (2012). Fractions: Could they really be the gatekeeper's doorman? Contemporary Educational Psychology, 31, 247-253. doi:10.1016/j.cedpsych.2012.07.001

Booth, J. L., \& Siegler, R. S. (2006). Developmental and individual differences in pure numerical estimation. Developmental Psychology, 41, 189-201. doi:10.1037/0012-1649.41.6.189

Booth, J. L., \& Siegler, R.S. (2008). Numerical magnitude representations influence arithmetic learning. Child Development, 79, 1016-1031. doi:10.1111/j.1467-8624.2008.01173.x

Burnham, K.P., \& Anderson, D.R. (2002). Model selection and multimodel inference: A practical information-theoretic approach (2nd edn.). New York: Springer.

Cohen, D. J., \& Blanc-Goldhammer, D. (2011). Numerical bias in bounded and unbounded number line tasks. Psychonomic Bulletin \& Review, 18, 331-338. doi:10.3758/s13423011-0059-z 
Ebersbach, M., Luwel, K., Frick, A., Onghena, P., \& Verschaffel, L. (2008). The relationship between the shape of the mental number line and familiarity with numbers in 5- to 9-yearold children: Evidence for a segmented linear model. Journal of Experimental Child Psychology, 99, 1-17. doi:10.1016/j.jecp.2007.08.006

Firebaugh, G. (2015). Statistics of ecological fallacy. In J. D. Wright (Ed.), International encyclopedia of the social and behavioral sciences (2nd ed., pp. 865-867). Oxford: Elsevier.

Heine, A., Thaler, V., Tamm, S., Hawelka, S., Schneider, M., Torbeyns, J., . . Jacobs, A. M. (2010). What the eyes already "know": Using eye movement measurement to tap into children's implicit numerical magnitude representations. Infant and Child Development, 19, 175-186. doi:10.1002/icd.640

Hurst, M., Leigh Monahan, K., Heller, E., \& Cordes, S. (2014). 123s and ABCs: Developmental shifts in logarithmic-to-linear responding reflect fluency with sequence values.

Developmental Science, 17, 892-904. doi:10.1111/desc.12165

Laski, E., \& Siegler, R. (2007). Is 27 a big number? Correlational and causal connections among numerical categorization, number line estimation, and numerical magnitude comparison. Child Development, 78, 1723-1743. doi:10.1111/j.1467-8624.2007.01087

Link, T., Huber, S., Nuerk, H.-C., \& Moeller, K. (2014). Unbounding the mental number line new evidence on children's spatial representation of numbers. Frontiers in Psychology, 4, 1021. doi:10.3389/fpsyg.2013.01021

Link, T., Nuerk, H.-C., \& Moeller, K. (2014). On the relation between the mental number line and arithmetic competencies. The Quarterly Journal of Experimental Psychology, 67, 1597-1613. doi:10.1080/17470218.2014.927515 
Luwel, K., Peeters, D., \& Verschaffel, L. (submitted). Developmental change in number line estimation: A strategy-based perspective. Manuscript submitted for publication.

Luwel, K., Peeters, D., Dierckx, G., Sekeris, E., \& Verschaffel, L. (2017). Raw data Luwel, Peeters, Dierckx, Sekeris, \& Verschaffel - NLE in atypical number lines - Can J Exp Psy [Data file]. Available from https://osf.io/gzytb/.

Newman, R. S., \& Berger, C. F. (1984). Children's numerical estimation: Flexibility in the use of counting. Journal of Educational Psychology, 76, 55-64. doi:10.1037/0022-0663.76.1.55

Opfer, J. E., Thompson, C. A., \& Kim, D. (2016). Free versus anchored numerical estimation: A unified account. Cognition, 149, 11-17. doi:10.1016/j.cognition.2015.11.015

Peeters, D., Degrande, T., Ebersbach, M., Verschaffel, L., \& Luwel, K. (2016). Children's use of number line estimation strategies. European Journal of Psychology of Education, 31, 117134. doi:10.1007/s10212-015-0251-Z

Peeters, D., Verschaffel, L., \& Luwel, K. (2017). Benchmark-based strategies in whole number line estimation. British Journal of Psychology, 108, 668-686. doi:10.1111/bjop.12233

Petitto, A. L. (1990). Development of number line and measurement concepts. Cognition and Instruction, 7, 55-78. doi:10.1207/s1532690xci0701_3

Reinert, R. M., Huber, S., Nuerk, H.-C., \& Moeller, K. (2015). Strategies in unbounded number line estimation? Evidence from eye-tracking. Cognitive Processing, 16, 359-363. doi:10.1007/s 10339-015-0675-z

Rouder, J. N., \& Geary, D. C. (2014). Children's cognitive representations of the mathematical number line. Developmental Science, 17, 525-536. doi:10.1111/desc.12166

Schneider, M., Heine, A., Thaler, V., Torbeyns, J., De Smedt, B., Verschaffel, L., . . Stern, E. (2008). A validation of eye movements as a measure of elementary school children's 
developing number sense. Cognitive Development, 23, 409-422.

doi:10.1016/j.cogdev.2008.07.002

Siegler, R. S., \& Booth, J. L. (2004). Development of numerical estimation in young children. Child Development, 75, 428-444. doi:10.1111/j.1467-8624.2004.00684.x

Siegler, R. S., \& Opfer, J. E. (2003). The development of numerical estimation: Evidence for multiple representations of numerical quantity. Psychological Science, 14, 237-243. doi:10.1111/1467-9280.02438

Simms, V., Clayton, S., Cragg, L., Gilmore, C., \& Johnson, S. (2016). Explaining the relationship between number line estimation and mathematical achievement: The role of visuomotor integration and visuospatial skills. Journal of experimental child psychology, 145, 22-33. doi: 10.1016/j.jecp.2015.12.004

Slusser, E. B., Santiago, R. T., \& Barth, H. C. (2013). Developmental change in numerical estimation. Journal of Experimental Psychology: General, 142, 193-208. doi:10.1037/a0028560

Sullivan, J., Juhasz, B., Slattery, T., \& Barth, H. (2011). Adults' number-line estimation strategies: evidence from eye movements. Psychonomic Bulletin \& Review, 18, 557-563. doi:10.3758/s13423-011-0081-1

White, S. L. J., \& Szucs, D. (2012). Representational change and strategy use in children's number line estimation during the first years of primary school. Behavioral and Brain Functions, 8, 1-12. doi:10.1186/1744-9081-8-1

Xu, C., \& LeFevre, J.-A. (2016). Training young children on sequential relations among numbers and spatial decomposition: Differential transfer to number line and mental transformation tasks. Developmental Psychology, 52, 854-866. doi:10.1037/dev0000124 


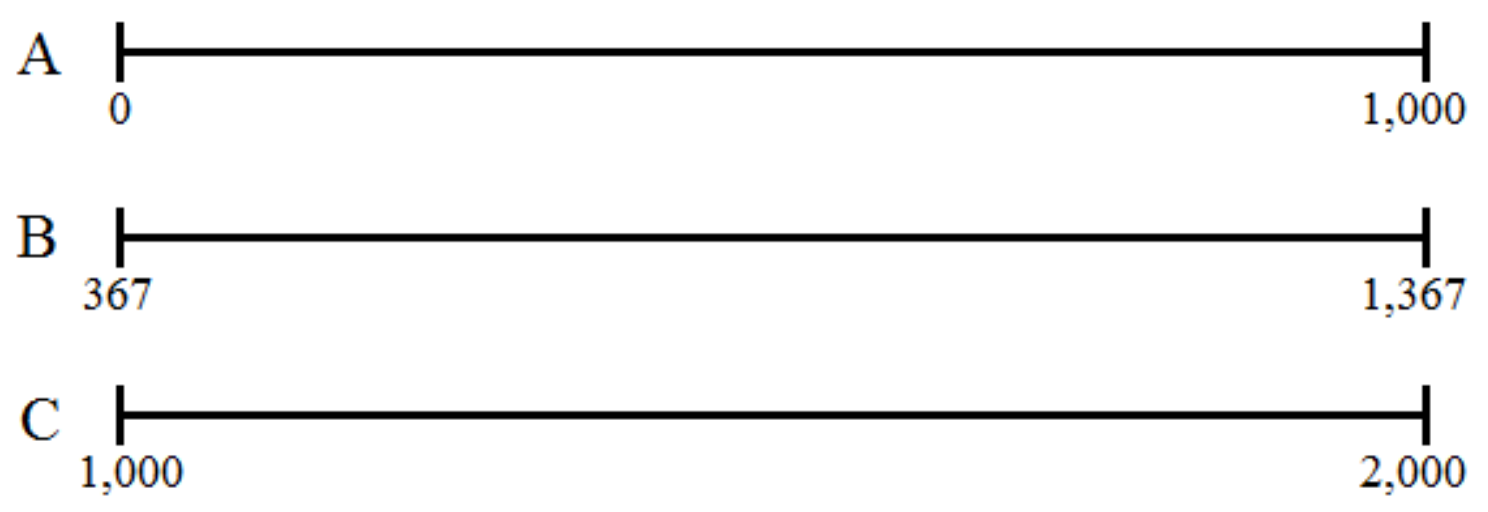

Figure 1. Presented number line in the (A) typical, (B) atypical, and (C) control condition. 


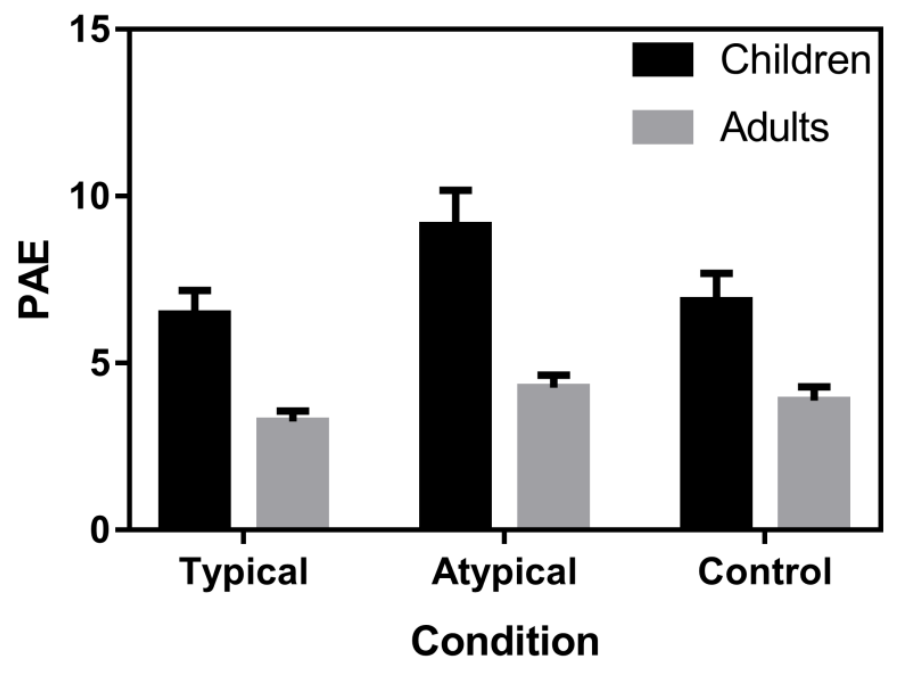

Figure 2. Mean \% of absolute error (and 95\% CI) for the typical, atypical, and control condition as a function of age. 


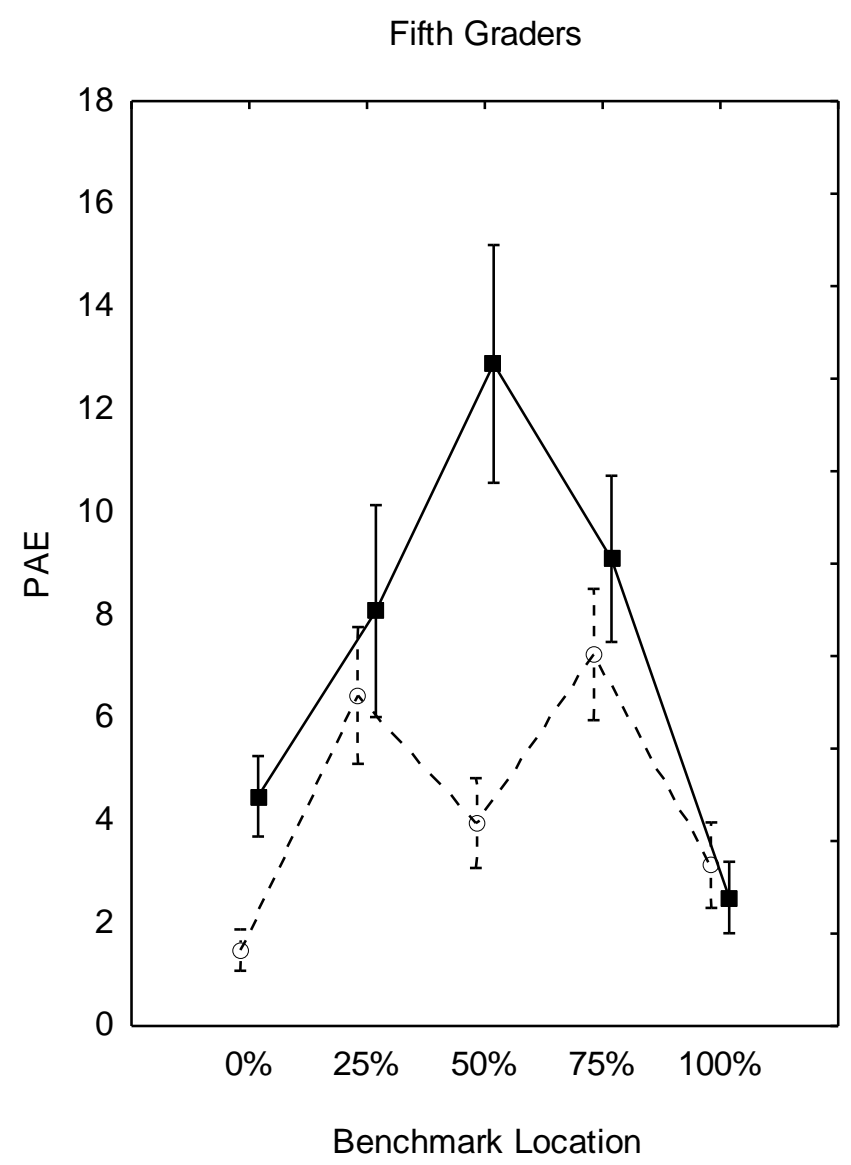

Adults

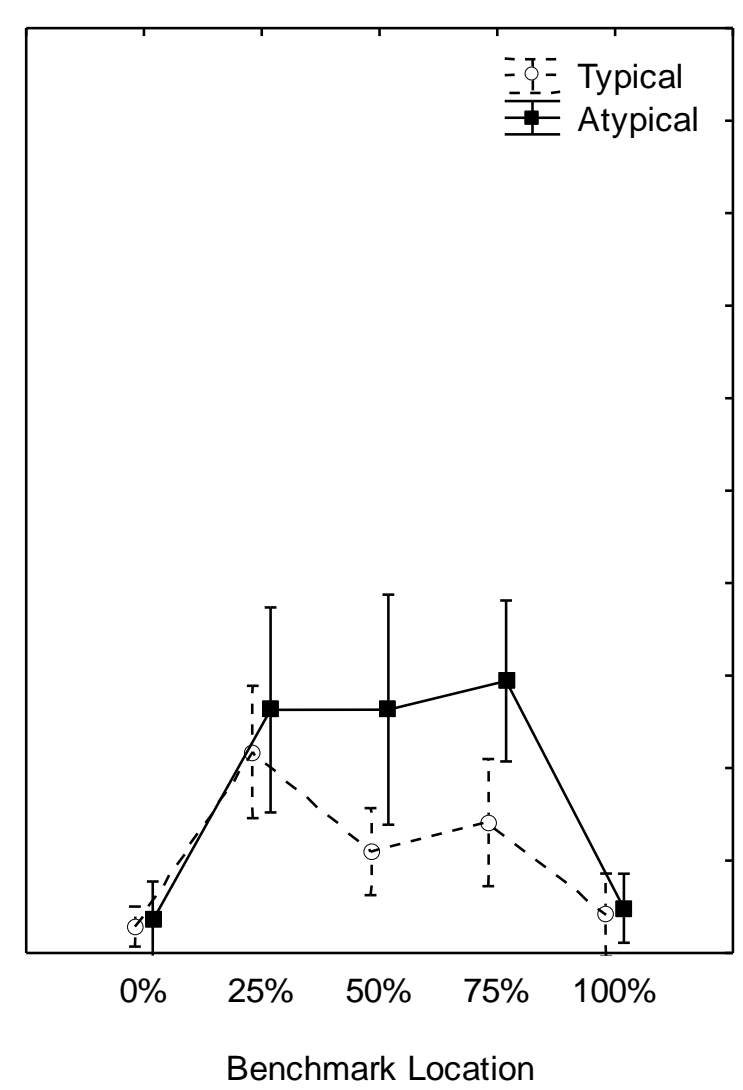

Figure 3. Mean \% of absolute error (and 95\% CI) at the benchmarks for the typical and atypical condition as a function of age. 
Table 1

Mean (and Standard Deviation of) Percentage of Absolute Error (PAE) in Fifth Graders and Adults as a function of Benchmark Location and Condition.

\begin{tabular}{|c|c|c|}
\hline \multirow[b]{2}{*}{ Benchmark location } & \multicolumn{2}{|c|}{ Condition } \\
\hline & Typical & Atypical \\
\hline & \multicolumn{2}{|c|}{ Grade 5} \\
\hline $0 \%$ & $1.48(1.51)$ & $4.47(3.05)$ \\
\hline $25 \%$ & $6.43(4.20)$ & $8.08(7.34)$ \\
\hline $50 \%$ & $3.95(3.30)$ & 12.89 (7.98) \\
\hline $75 \%$ & 7.23 (4.676) & $9.09(5.23)$ \\
\hline \multirow[t]{2}{*}{$100 \%$} & $3.13(3.23)$ & $2.50(2.59)$ \\
\hline & \multicolumn{2}{|c|}{ Adults } \\
\hline $0 \%$ & $0.51(0.45)$ & $0.64(0.45)$ \\
\hline $25 \%$ & $3.92(3.03)$ & $4.74(3.36)$ \\
\hline $50 \%$ & $1.98(0.98)$ & $4.73(4.26)$ \\
\hline $75 \%$ & $2.54(1.81)$ & $5.29(3.52)$ \\
\hline $100 \%$ & $0.74(0.51)$ & $0.87(0.86)$ \\
\hline
\end{tabular}


Table 2

Proportion of Children's and Adults' Estimation Patterns best fit by the One-, Two-, and FourCycle Power Model in the Typical and Atypical Condition

\begin{tabular}{cccccccc}
\hline & \multicolumn{3}{c}{ Children } & & \multicolumn{3}{c}{ Adults } \\
\cline { 2 - 3 } Condition & 1-cycle & 2-cycle & 4-cycle & & 1-cycle & 2-cycle & 4-cycle \\
Typical & .53 & .37 & .10 & & .28 & .09 & .63 \\
Atypical & .73 & .17 & .10 & & .44 & .25 & .31 \\
\hline
\end{tabular}




\section{Appendix 1}

Matched Target (top) and Practice Numbers (bottom) for the Typical, Atypical, and Control Condition

\begin{tabular}{|c|c|c|c|}
\hline $\begin{array}{l}\text { Position on } \\
\text { number line }\end{array}$ & Typical & Atypical & Control \\
\hline$\underline{0.5}$ & 5 & 372 & 1004 \\
\hline 6.9 & 69 & 436 & 1068 \\
\hline 12.3 & 123 & 490 & 1122 \\
\hline 19 & 190 & 557 & 1191 \\
\hline$\underline{24.6}$ & 246 & 613 & 1247 \\
\hline 27.1 & 271 & 638 & 1270 \\
\hline 33.7 & 337 & 704 & 1336 \\
\hline 38.4 & 384 & 751 & 1385 \\
\hline 42.2 & 422 & 789 & 1423 \\
\hline 45.8 & 458 & 825 & 1459 \\
\hline$\underline{50.2}$ & 502 & 869 & 1503 \\
\hline 57.3 & 573 & 940 & 1572 \\
\hline 61.6 & 616 & 983 & 1617 \\
\hline 66.4 & 664 & 1031 & 1665 \\
\hline 71 & 710 & 1077 & 1711 \\
\hline$\underline{75.5}$ & 755 & 1122 & 1754 \\
\hline 83.9 & 839 & 1206 & 1838 \\
\hline 88.8 & 888 & 1255 & 1889 \\
\hline 94.1 & 941 & 1308 & 1940 \\
\hline$\underline{99.7}$ & 997 & 1364 & 1996 \\
\hline 40.8 & 408 & 775 & 1408 \\
\hline 85.1 & 851 & 1218 & 1851 \\
\hline
\end{tabular}

Note. Underlined positions are target numbers that were intentionally located close to the position of a potential benchmark (i.e., at $0,25,50,75$, and $100 \%$ of the number line). 


\section{Appendix 2}

$N$-cycle power functions

To position a given target number $x$ on a number line bounded by two benchmarks $b_{\min }$ and $b_{\max }$, the participant assesses the proportion $p=\left(x-b_{\min }\right) /\left(b_{\max }-b_{\min }\right)$. According to proportion judgment models, the target number $x$ is estimated by $x_{\min }+p\left(x_{\max }-x_{\min }\right)$. The value $p$ determines the proportion $p=\left(x-b_{\min }\right) /\left(b_{\max }-b_{\min }\right)$ of the smaller magnitude $x-b_{\min }$ to the larger magnitude $b_{\max }-b_{\min }$. According to Stevens' law the smaller magnitude can be estimated by $\alpha_{1}$ $\left(x-b_{\min }\right)^{\beta}$. Since the larger magnitude $b_{\max }-b_{\min }$ can be seen as the sum of $\left(x-b_{\min }\right)$ and $\left(b_{\max }-\right.$

$x$, Stevens' power law results in an estimate of $\alpha_{1}\left(x-b_{\min }\right)^{\beta}+\alpha_{2}\left(x-b_{\min }\right)^{\beta}$. Finally, with $\alpha=\alpha_{2}$ $/ \alpha_{1}$

$$
\mathrm{p}=\alpha_{1}\left(x-\mathrm{b}_{\min }\right)^{\beta} /\left(\alpha_{1}\left(x-\mathrm{b}_{\min }\right)^{\beta}+\alpha_{2}\left(b-\mathrm{x}_{\min }\right)^{\beta}\right)=\left(x-\mathrm{b}_{\min }\right)^{\beta} /\left(\left(x-\mathrm{b}_{\min }\right)^{\beta}+\alpha\left(b-\mathrm{x}_{\min }\right)^{\beta}\right)
$$

Often $\alpha=\alpha_{2} / \alpha_{1}$ is assumed to be equal to 1 . In the present study, we have weakened this assumption as will be illustrated for the one-cycle model below.

\section{One-cycle model}

To position a given target number $x$ on a number line bounded by two benchmarks $b_{0}$ and $b_{100 \text {, }}$ the abovementioned proportion judgement strategy is applied for $b_{\min }=b_{0}$ and $b_{\max }=b_{100}$. As such, the target number $x$ is estimated by

$$
\mathrm{b}_{0}+\left(\mathrm{b}_{100}-\mathrm{b}_{0}\right)\left(x-\mathrm{b}_{0}\right)^{\beta} /\left(\left(x-\mathrm{b}_{0}\right)^{\beta}+\alpha\left(\mathrm{b}_{100}-\mathrm{x}\right)^{\beta}\right)
$$

As mentioned before, regular one-cycle models assume that $\alpha$ equals 1. Figure 1 illustrates that the predictions in a NLE task by using the abovementioned regular one-cycle model with $\alpha$ equals 1 show a $S$ pattern (or inverse $S$ pattern - depending the value for $\beta$ ) with central symmetry. The central symmetry stems from the fact that to determine the larger magnitude $b_{\max }-b_{\min }$, the same scaling factor and the same exponent is used to estimate $\left(b_{\max }-x\right)$ 
and $\left(x-b_{\min }\right)$. As such participants are assumed to treat distances from the begin point in the same way as distances from the end point.

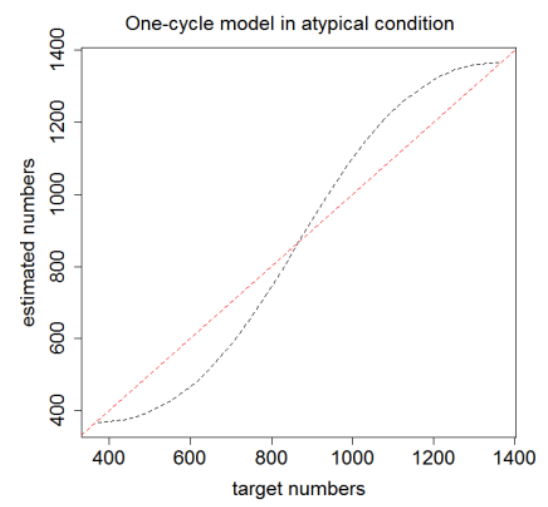

Figure 1: black dotted line : predictions in a NLE task where participants are asked to position a target number $\mathrm{x}$ on an atypical range 367 - 1,367 using a regular one-cycle model $(\alpha=1$ and $\beta=1.86$.$) . Red dotted line: indicates a perfect estimation pattern.$

In Figure 2(a) we see the estimation pattern of a fifth-grade child (id 53) that clearly does not show central symmetry in the atypical condition. To account for this, in the present study we allow for both age groups and conditions the scaling factor $\alpha$ to be different from 1 . In Figure 2(a) a fit using the one-cycle model $\alpha=0.35$ and thus different from 1 (full line) is compared to the fit by the regular one-cycle model with $\alpha=1$ (dotted line).
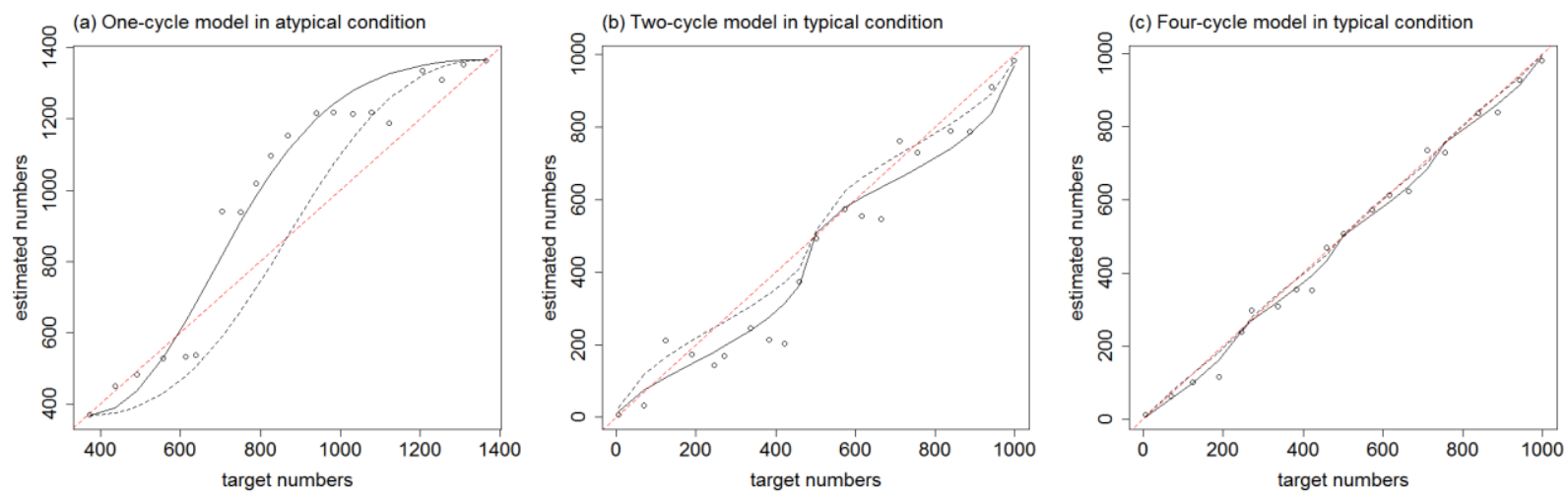

Figure 2a: (a) Estimated numbers using a one cycle model for a fifth-grade child in the atypical condition with $\beta=1.86$; full line : $\alpha=0.35$; dotted line: $\alpha=1$. (b) Estimated numbers using 
a two cycle model for a fifth-grade child in the typical condition with $\beta=0.64$; full line: $\alpha=1.73$; dotted line: $\alpha=1$. (c) Estimated numbers using a four-cycle model for an adult with $\beta=0.86$; full line : $\alpha=1.47$; dotted line: $\alpha=1$

\section{Two-cycle model}

The two-cycle model can be used when a participant uses the begin point $b_{0}$, the midpoint $b_{50}$ and the endpoint $b_{100}$ as benchmarks. The number line can be seen as a sequence of two smaller number lines $b_{0}-b_{50}$, and $b_{50}-b_{100}$. On each of the smaller number lines, the abovementioned proportion judgement strategy can be applied. In case the target number $x$ is smaller than the midpoint, the participant can apply the proportion judgement strategy on the first half of the number line by using the begin point for $b_{\min }$ and the midpoint for $b_{\max }$. In case the target number $x$ is larger than the midpoint, the participant can apply the proportion judgement strategy on the number line ranging from $b_{50}$ to $b_{100}$. This results in the following estimate for the target number $x:$

$$
\begin{array}{ll}
\text { if } x<b_{50}: & b_{0}+\left(b_{50}-b_{0}\right)\left(x-b_{0}\right)^{\beta} /\left(\left(x-b_{0}\right)^{\beta}+\alpha\left(b_{50}-x\right)^{\beta}\right) \\
\text { if } x>b_{50}: & b_{50}+\left(b_{100}-b_{50}\right)\left(x-b_{50}\right)^{\beta} /\left(\left(x-b_{50}\right)^{\beta}+\alpha\left(b_{100}-x\right)^{\beta}\right)
\end{array}
$$

Again often $\alpha$ is assumed to be equal to 1 . In Figure 2 (b), the two-cycle model with $\alpha=1$ and $\alpha$ different from 1 is illustrated for the same child as in Figure 2(a) (id nr 53) but now under the typical condition.

\section{Four-cycle model}

The four-cycle model can be used when a participants also use the two quartiles $b_{25}$ and $b_{75}$ as benchmarks. The number line can be seen as a sequence of four smaller number lines $b_{0}-b_{25}$, $b_{25}-b_{50}, b_{50}-b_{75}$ and $b_{75}-b_{100}$. On each of the smaller number lines, the abovementioned proportion jugdgement strategy can be applied. For the first smaller number line, in case the target number $x$ is smaller than the first quartile, the participant can apply the proportion 
judgement strategy using the begin point $b_{0}$ for $b_{\min }$ and using the first quartile $b_{25}$ for $b_{\max }$. An analoguous approach can be followed for the other three smaller number lines.

$$
\begin{array}{ll}
\text { if } \mathrm{b}_{0}<x<\mathrm{b}_{25}: & \mathrm{b}_{0}+\left(\mathrm{b}_{25}-\mathrm{b}_{0}\right)\left(x-\mathrm{b}_{0}\right)^{\beta} /\left(\left(x-\mathrm{b}_{0}\right)^{\beta}+\alpha\left(\mathrm{b}_{25}-x\right)^{\beta}\right) \\
\text { if } \mathrm{b}_{25}<x<\mathrm{b}_{50}: & \mathrm{b}_{25}+\left(\mathrm{b}_{50}-\mathrm{b}_{25}\right)\left(x-\mathrm{b}_{25}\right)^{\beta} /\left(\left(x-\mathrm{b}_{25}\right)^{\beta}+\alpha\left(\mathrm{b}_{50}-x\right)^{\beta}\right) \\
\text { if } \mathrm{b}_{50}<x<\mathrm{b}_{75}: & \mathrm{b}_{50}+\left(\mathrm{b}_{75}-\mathrm{b}_{50}\right)\left(x-\mathrm{b}_{50}\right)^{\beta} /\left(\left(x-\mathrm{b}_{50}\right)^{\beta}+\alpha\left(\mathrm{b}_{75}-x\right)^{\beta}\right) \\
\text { if } \mathrm{b}_{75}<x<\mathrm{b}_{100:} & \mathrm{b}_{75}+\left(\mathrm{b}_{100}-\mathrm{b}_{75}\right)\left(x-\mathrm{b}_{75}\right)^{\beta} /\left(\left(x-\mathrm{b}_{75}\right)^{\beta}+\alpha\left(\mathrm{b}_{100}-x\right)^{\beta}\right)
\end{array}
$$

In Figure 2 (c), the four-cycle model is illustrated for an adult (id $\mathrm{nr} 2$ ) in the typical condition.. 


\section{Appendix 3}

\section{Median-based model fittings}

Table A.1

AICc (and $\triangle A I C$ ) for the Fittings of the Different N-cycle Power Models on the Median Estimates of the Fifth Graders and the Adults in the Typical and Atypical Condition

\begin{tabular}{|c|c|c|}
\hline \multirow[b]{2}{*}{ Model } & \multicolumn{2}{|c|}{ Condition } \\
\hline & Typical & Atypical \\
\hline & \multicolumn{2}{|c|}{ Grade 5} \\
\hline 1-cycle & $137.7(4.1)$ & $154.0(9.1)$ \\
\hline 2-cycle & 125.9 & 144.9 \\
\hline \multirow[t]{2}{*}{ 4-cycle\% } & $142.0(16.1)$ & $152.2(7.3)$ \\
\hline & \multicolumn{2}{|c|}{ Adults } \\
\hline 1-cycle & $120.5(7.9)$ & $114.7(2.4)$ \\
\hline 2-cycle & $118.8(6.2)$ & 112.3 \\
\hline 4-cycle & 112.6 & $119.8(7.5)$ \\
\hline
\end{tabular}

Note. Statistically preferred model (as determined by lowest AICc) in bold. All other models are tested for support by calculating the $\triangle \mathrm{AICc}$ with the preferred model.

Fitting a one-, a two-, and a four-cycle power model to the median estimates of children and adults for the typical and atypical condition revealed that the median estimates of the fifth graders 
were, best described by a 2-cycle power model in both conditions (see Table A.1), suggesting that fifth graders made use of the midpoint in both the typical as well as the atypical condition. The median estimates of the adults were best described by a 4-cycle power model in the typical condition, reflecting the use of the midpoint and the quartiles, and by a 2-cycle power model in the atypical condition. 